\title{
Avaliação na Aprendizagem Baseada em Problemas (ABP): Reflexões acerca do processo
}

\begin{abstract}
Fabrício Luz Cardoso', Sílvia Franco da Rocha Tonhom², José Antonio Galbiatti ${ }^{2}$ e Mara Quaglio Chirelli ${ }^{2}$

${ }^{1}$ Estudante da Faculdade de Medicina de Marília, Brasil | fabricioramalhense@gmail.com | https://orcid.org/ 0000-0000-0000-0000

${ }^{2}$ Faculdade de Medicina de Marília, Brasil | siltonhom@gmail.com; galbiatti.mao@gmail.com; marachirelli@gmail.com | http://orcid.org/-0000-0001-7522-2861; https://orcid.org/0000-0003-2330-3816 ;https://orcid.org/0000-0002-7417-4439

Resumo: Introdução: A Aprendizagem Baseada em Problemas (ABP) ou Problem based learning (PBL) é um método ativo, entre outros, utilizado por instituições de ensino superior e a avaliação é um aspecto fundamental do processo de ensino-aprendizagem. Considerando o tempo de desenvolvimento de métodos ativos, identificou-se a necessidade de realizar uma investigação em uma faculdade do interior paulista/Brasil, com o objetivo de analisar como está sendo desenvolvido o processo de avaliação, no cenário de tutorial dessa instituição. Método: fundamentou-se de acordo com a metodologia de pesquisa qualitativa em saúde com a realização de entrevistas com trinta estudantes da $1^{\circ}$ a $4^{\circ}$ série de medicina e $1^{\circ}$ e $2^{\circ}$ séries de enfermagem da referida instituição, no ano de 2018 e com dezoito tutores com no mínimo dois anos de experiência. Utilizou-se um instrumento com uma parte de identificação e outra com questões norteadoras. A análise dos dados foi facilitada pelo uso do software de análise qualitativa webQDA® e orientada pela análise de conteúdo temática. Resultados: Após o processamento da análise, identificou-se alguns temas, cujo aspectos referente a Avaliação será explorado neste artigo: compreensões acerca das avaliações durante o processo tutorial; critérios de avaliação e dificuldade em avaliar o desempenho de estudantes e professores. Conclusão: A abordagem qualitativa possibilitou analisar o que estudantes e docentes da instituição pesquisada pensam a respeito da estrutura da avaliação no PBL e permitiu a observação das experiências de outras instituições de ensino superior ao redor do mundo.
\end{abstract}

Palavras-chave: Aprendizagem Baseada em Problemas; Avaliação; Ensino; Enfermagem Medicina.

\begin{abstract}
Assessment in Problem-Based Learning (PBL): Reflections on the Process
Abstract: Introduction: Problem Based Learning (PBL) or Problem based learning (PBL) is an active method, among others, used by higher education institutions and assessment is a fundamental aspect of the teaching-learning process. Considering the time of development of active methods, the need to carry out an investigation in a college in the interior of São Paulo / Brazil was identified, with the aim of analyzing how the evaluation process is being developed, in the tutorial scenario of that institution. Method: it was based on the qualitative research methodology in health with interviews with thirty students from the 1st to the 4th series of medicine and the 1st and 2nd series of nursing from that institution, in the year 2018 and with eighteen tutors with at least two years of experience. An instrument with an identification part and another with guiding questions was used. Data analysis was facilitated by the use of qualitative analysis software webQDA® and guided by thematic content analysis. Results: After processing the analysis, some themes were identified, whose aspects regarding the Evaluation will be explored in this article: understandings about the evaluations during the tutorial process; evaluation criteria and difficulty in evaluating the performance of students and teachers. Conclusion: The qualitative approach made it possible to analyze what students and professors of the researched institution think about the structure of the evaluation in the PBL and allowed the observation of the experiences of other institutions of higher education around the world.
\end{abstract}

Keywords: Problem-based Learning; Assessment; Teaching; Nursing; Medicine.

\section{Introdução}

Medicina e Enfermagem são áreas em constante metamorfose acompanhando as tendências, inovações/tecnologias, exigências e realidades da contemporaneidade. As instituições de ensino superior encarregadas pela formação dos profissionais de saúde são desafiadas, a responder às demandas sociais e se atualizar com relação às propostas educacionais em suas escolas de ciencias da saúde (Moraes \& Manzini, 2006). 
O Brasil conduziu uma mudança em sua filosofia de atenção à saúde populacional com a substituição do modelo hospitalocêntrico e curativo para um modelo pautado nas esferas biopsicossocioambientais do indivíduo, cuja gênese da transformação se deu com a criação do Sistema Único de Saúde (SUS) em 1988 (Moraes \& Manzini, 2006). Para tal, necessita-se de investimentos na formação professional. Internacionalmente, a temática recebeu notoriedade em eventos como a Conferência de Edimburgo, o Encontro Internacional de Educação Médica e o programa da OMS voltado para a transformação da educação médica (Oliveira et al, 2008).

O PBL - Problem based learning ou ABP - Aprendizagem baseada em problemas, destaca como uma Metodologia Ativa de ensino-aprendizagem, sendo precursora no curso de medicina da Universidade de McMaster (Canadá) na década de 60 (Spaulding, 1969). No Brasil o PBL/ABP chegou às universidades na segunda metade da década de 90, sendo como pioneira a Faculdade de Medicina de Marília, em 1997 (Ribeiro \& Mizukami, 2004).

$\mathrm{Na}$ instituição do estudo, a estrutura curricular está organizada em séries anuais e segue a um tripé composto por três unidades: Unidade de Prática Profissional (UPP), Unidade Educacional Sistematizada (UES) e Unidade Educacional Eletiva (UEE), desenvolvida a partir da segunda série (Faculdade de Medicina de Marília, 2014)

O processo tutorial do PBL envolve a leitura atenta dos casos, elaboração de hipóteses, realização da "Tempestade de idéias", elaboração das questões de aprendizagem, momento de estudo individual, discussão em grupo dos conteúdos estudados individualmente, avaliação de si, do grupo e do tutor ao final de cada encontro e, considerando, que o professor exerce função importante de mediador, é necessário que esse processo seja compreendido e aprimorado a cada vez mais (Komatsu et al, 2000).

A avaliação é parte imprescindível do processo de ensino-aprendizagem. Para que seja precisa e completa, se faz necessária a obtenção de informações a partir de situações e fontes diversificadas. (Depresbiteris, 2001).

Perrenoud, (1999) relata dois fundamentos na avaliação: a avaliação formativa e a avaliação somativa.

A avaliação quando formativa indica que o ato de avaliar não faz sentido se isolado, ele deve ser constituinte de todo um processo de ensino-aprendizagem no qual a avaliação não é mais pontual e sim contínua (Reid, 2011). Nessa perspectiva, todas as informações produzidas pela integração e interação entre professores e estudantes, bem como entre os estudantes, são pertinentes (Reid, 2011).

Diante desse contexto, nota-se a necessidade da realização de estudos que permitam elucidar como está sendo desenvolvida a avaliação no processo de ensinoaprendizagem do PBL. Assim, esse estudo objetivou analisar o que estudantes e docentes de uma instituição do interior paulista/Brasil pensam a respeito da estrutura da avaliação no PBL nessa instituição.

\section{Metodologia}

Este estudo caracteriza-se como uma pesquisa descritiva de natureza qualitativa. De acordo com Minayo et al (2016) a pesquisa qualitativa ocupa um avançado nível de realidade no campo das ciências sociais, pois, responde as questões de maneira singularizada, lida com a totalidade das causas, dos significados, das aspirações, das convicções, das atitudes e dos valores do ser humano, que são parte da realidade social.

A pesquisa teve como cenário a Unidade Educacional Sistematizada (UES) das $1^{\circ}, 2^{\circ}, 3^{\circ}$ e $4^{\circ}$ séries de medicina e $1^{\circ}$ e $2^{\circ}$ séries de enfermagem de uma faculdade do interior paulista/Brasil. No modelo educacional proposto (PBL/ABP) a UES é responsável pela maior parte do conhecimento teórico adquirido nas séries, conhecimento este relativo às disciplinas e habilidades esperadas, que estão envolvidas nos casos de tutoria, descritas no manual de série de cada série. 
Portanto, pressupõe-se que uma boa condução da UES pelo tutor e um bom aproveitamento por parte dos estudantes torna o processo tutorial mais eficaz.

Os critérios de inclusão na investigação foram: (a) Ser acadêmico ou professor tutor de uma das séries de medicina $\left(1^{\circ}, 2^{\circ}, 3^{\circ}\right.$ e $\left.4^{\circ}\right)$ ou de enfermagem $\left(1^{\circ}\right.$ e $\left.2^{\circ}\right)$ da faculdade em questão, no período de 2017-2018, (b) Concordar com o estudo assinando o termo de livre esclarecimento; (c) Desenvolver atividades de tutoria há pelo menos dois anos (somente para professores), tais critérios condizem com o cenário de estudo que é o grupo de tutoria e foram escolhidas as séries citadas por serem séries onde o processo tutorial acontece seguindo os passos da aprendizagem baseada em problemas, permitindo maior contato entre tutores e alunos. Contou-se, na totalidade, com 20 estudantes de medicina e 10 estudantes de enfermagem distribuídos nas séries; e 12 docentes da medicina e 6 docentes da enfermagem. Estudantes e professores foram distribuídos entre os 40 grupos de tutoria, garantindo, assim, a homogeneidade amostral.

Antes da coleta de dados, os participantes leram e assinaram o Termo de Consentimento Livre e Esclarecido (TCLE). As entrevistas foram realizadas e transcritas pelo pesquisador. Utilizou-se um instrumento para desenvolvimento das entrevistas, tanto para professores como para os estudantes, organizado de forma semi-estruturado contendo dados de identificação e questões norteadoras abordando o objeto de estudo.

Para a análise dos dados, optou-se pela modalidade de Análise Temática de conteúdo, que segundo Minayo et al. (2016) é a mais apropriada quando se faz uso da investigação qualitativa em saúde.

Como ferramenta de apoio na análise temática utilizou-se o software de análise qualitativa webQDA®.

A análise temática baseia-se na descoberta dos núcleos de sentido constituintes de uma comunicação, na qual a presença é importante para a compreensão do objeto de análise do estudo. É dividida em três etapas: (1) pré-análise, (2) exploração do material e (3) tratamento dos resultados obtidos e interpretação (Minayo et al2016).

Na pré-análise realizou-se a leitura flutuante e exaustiva das entrevistas, constituição do corpus, buscando captar as ideias centrais de estudantes e professores acerca do processo de avaliação. Na exploração do material foi selecionado os fragmentos das entrevistas e, em seguida, foi identificado os núcleos de sentido e agrupados em temáticas para posterior análise.

Para manter $\mathrm{O}$ anonimato, os participantes foram identificados com a letra $\mathrm{E}$ para estudantes e P para professor, seguido de ordem numérica conforme realização das entrevistas.

Este projeto de pesquisa foi aprovado pelo CEP - Comitê de Ética em Pesquisa (envolvendo seres humanos) sob o parecer consubstanciado $n^{\circ} 2.300 .019$.

\section{Resultados}

As entrevistas revelaram um eixo-temático relacionados à avaliação no $\mathrm{PBL}$ depreendido após transcrição e categorização das mesmaa, utilizando-se o webQDA® - software de análise qualitativa que o nomeia como código-árvore. A seguir serão apresentados os resultados conforme os temas extraídos.

\subsection{Compreensões acerca das Avaliações Durante o Processo Tutorial}

Durante o processo tutorial estudantes e tutores relataram que realizam avaliação em todos os momentos, sendo que o professor estimula que o estudante o realize com todos os membros do grupo, conforme exemplificado nos fragmentos a seguir: 
"A gente geralmente realiza a avaliação no final de todas as sessões de tutoria, de abertura de caso, discussão e fechamento" [E-22].

"A gente realiza, todo mundo no final da discussão (...) toda tutoria a gente faz a avaliação tanto dos membros, quanto do tutor" [E-24].

"Então, todo final de tutoria eu faço avaliação estimulando o estudante a fazer a sua auto avaliação, avaliação dos colegas dele e a minha avaliação, porque eu também não sou perfeito obviamente, então eu sempre deixo aberto a eles que eu sou muito aberto a críticas até pra mudar alguns desempenhos e algum comportamento" [T- 47].

$\mathrm{Na}$ avaliação realizada, tutores e estudantes ressaltam a importância de abordarem das relações interpessoais, visto que o processo é desenvolvido em grupo.

"Então é para além de simplesmente seguir os passos da tutoria e obviamente também estar atento as relações no grupo porque é um aspecto importante que também interfere na construção do conhecimento no processo ensino-aprendizagem, as relações do grupo" [T-33].

"a gente costuma fazer as críticas quando necessárias de um forma polida, a gente não esquece, a gente não fica só no elogio a gente tenta criticar de uma forma polida também" [E-21].

\subsection{Critérios de Avaliação}

Em relação aos critérios utilizados para o desenvolvimento do processo de avaliação, os participantes citaram aspectos individuais e do movimento do grupo, conforme fragmentos a seguir:

"O principal critério era a participação, participou mais, participou menos tal" [E-6].

"A gente também gostava muito de avaliar um ao outro, porque tipo todo mundo recebia muito bem, então isso foi até positivo para as pessoas que eram mais tímidas, ter esse feedback do outro era importante" [E-13].

"Os critérios de colaboração, do ponto de partida e ponto de chegada do estudante, porque ele começa de uma forma e pode terminar de outra e eu tenho que acompanhar e mostrar isso para ele. Eu tento valorizar muito desde as pequenas coisas, a contribuição, o respeito com os colegas, respeito com tutor, respeitar o jeito de cada um" [T-34].

\subsection{Dificuldade em Avaliar o Desempenho de Estudantes e Professores}

Nessa pesquisa foi notória a identificação de que a avaliação é imprescindível ao processo, que existem iniciativas de realização, contudo, existem fragilidades para o seu desenvolvimento tanto por parte de professores quanto de estudantes, como pode-se verificar nos fragmentos a seguir:

"Eu sou uma pessoa muito tímida e ele [tutor] não soube falar comigo, era o começo do primeiro ano e na avaliação eu não sabia nem o que falar direito, tava começando, e eu falei: ah eu estudei bastante para esse problema, ai ele chegou e falou: se você estudou bastante porque você não acrescentou nada de interessante na discussão de hoje? Isso pra mim, tipo eu me inibi em vez de participar mais, eu tinha medo do meu tutor (...) [E-15].

"O meu tutor já falou pra gente que ele tem uma certa dificuldade em avaliar os pontos positivos, ele foca só nos negativos e eu acho que isso é um problema, (...), não destaca o avanço que as vezes a pessoa teve" [E-28]. 
"Só que eu acho que existe ainda uma dificuldade muito grande de avaliar quando são os aspectos negativos, os positivos eles fluem muito mais fácil, mas apontar uma coisa que não ta muito bem as vezes precisa de um empurrão do tutor" [T-50].

Evidenciou-se também dificuldades em realizá-las, em especial, com menos aprofundamento nos anos seguintes, conforme depoimentos a seguir:

"Sim. No quarto ano eu considero que as avaliações são bem menores do que no primeiro e segundo ano. No quarto ano se ele tá mais preocupado em fazer avaliações técnicas, então dificilmente, assim é duro você trazer o grupo pra fazer uma avaliação individual e mesmo quando feitas elas são bem pontuais, não tem aquela avaliação mais geral do que é feito no primeiro, segundo e terceiro ano onde eu já fui tutor" [T-49].

"É uma avaliação que fica meio aberta também, não é muito objetiva e detalhada, mas sempre tem avaliação, a tutoria foi boa, eu participei bem, todo mundo contribuiu, o tutor participou, esse tipo de avaliação" [T-41].

\section{Discussão}

A avaliação proposta pela instituição expressa a importância de considerar o movimento do processo tutorial, permitindo a autoavaliação do estudante e do tutor; a avaliação individual dos pares e do tutor por parte dos estudantes; a avaliação do grupo como um todo pelos estudantes e tutor; a avaliação individual dos estudantes por parte do tutor e as avaliações dos casos de tutoria e pontuação das dificuldades encontradas no estudo. $\mathrm{Na}$ essência do PBL/ABP tal momento avaliativo deve ser realizado a fim de toda sessão de tutoria (Komatsu, 1999; Zanolli et al, 2002).

Nota-se que alguns grupos seguem com afinco a proposta de avaliação da escola, outros se utilizam somente de alguns pontos e outros não a valorizam.

A principal hipótese aventada para discrepância entre a forma com que a avaliação é realizada nos mais variados grupos dos cursos de medicina e enfermagem se deve, na opinião dos entrevistados, às dificuldades institucionais com capacitação de tutores e cotutores, hipótese esta também mencionada amplamente na literatura de educação médica (Komatsu, 1999; Faria et al, 2008).

No tocante aos critérios de avaliação, tutores e estudantes trouxeram em suas falas que geralmente se avalia: a participação dos estudantes, postura do estudante na tutoria e interação com o grupo, qualidade e diversidade das fontes de pesquisa, pontualidade, assiduidade.

Evidenciou dificuldades de estudantes e tutores em realizar avaliação de pontos negativos e também receber avaliação negativa. É notório que muitas vezes a repulsa pela crítica proferida está ligada à forma com que esta crítica é realizada, tornando-se o momento avaliativo promotor de inibição e resistência progressivas por parte da pessoa avaliada.

Barreto et al, (2017) encontraram aspectos semelhantes na avaliação realizada por estudantes e tutores em sessão de tutoria do curso médico. Destacam-se: I) para os estudantes entrevistados certas dificuldades dos tutores estão relacionadas a algumas características pessoais, como relutância em enfrentar o outro, fazer e receber críticas ou simplesmente não gostar/sentir prazer em avaliar; II) estudantes relatam apresentar algum "sentimento aversivo", como medo, ansiedade e frustração, durante as avaliações nas sessões tutoriais; III) a falta de maturidade dos estudantes durante essas avaliações é evidenciada pela dificuldade de enfrentar uma nova forma de avaliar, quando os estudantes veem como perseguição a crítica elaborada, constatando conflitos, atritos e mesmo brigas durante esse momento. 
Seren \& Ustun, (2008) avaliaram estudantes de duas escolas, sendo uma com método tradicional e outra com método PBL. Os escores de habilidades de resolução de conflitos e subescala (empatia, habilidades de escuta, abordagem baseada em requisitos, adaptação social e controle da raiva) formados por ABP foram significativamente maiores do que aqueles educados pelo método convencional de educação. Isso sugere que a ênfase nas habilidades interpessoais, na comunicação em equipe e na autoconsciência que fazem parte do currículo do ABP também deve se tornar um foco nos currículos convencionais. Essas mesmas competências e habilidades devem ser mais proeminentes nas escolas convencionais de enfermagem à medida que se tornam mais focadas no estudante.

\section{Conclusão}

A pesquisa com abordagem qualitativa alcançou sua proposta de conhecer o que estudantes e docentes da instituição pensam a respeito da avaliação realizada durante 0 processo tutorial.

As compreensões acerca da avaliação vão ao encontro da proposta institucional, cuja intenção é proporcionar o conhecimento da implementação do processo tutorial, possibilitando identificar as fragilidades para busca de superação entre os desempenhos de estudantes e professores. Contudo, ainda, identifica-se, muitos desafios!

Destaca-se, a diversidade de critérios estabelecidos, dificultando uma avaliação mais objetiva e podendo captar a singularidades dos estudantes, além de limítes de professores e estudantes quanto ao aprofundamento dos processos avaliativos, de forma a articuladar os aspectos cognitivo, afetivo e psicomotor, buscando o alcance do desempenho esperado.

Nesse sentido, investimentos em capacitação docente quanto a proposta curricular e, em especial, o processo de avaliação se fazem necessário.

\section{Agradecimentos}

À Fundação de Amparo a Pesquisa do Estado de São Paulo (FAPESP) por financiar o desenvolvimento da pesquisa por meio do Processo no 17/19474-3.

\section{Referências}

Moraes, M. A. A. D., \& Manzini, E. J. (2006). Concepções sobre a aprendizagem baseada em problemas: um estudo de caso na Famema. Revista Brasileira de Educação Médica, 30(3), 125-135.

Oliveira, N. A. D., Meirelles, R. M. S. D., Cury, G. C., \& Alves, L. A. (2008). Mudanças curriculares no ensino médico brasileiro: um debate crucial no contexto do Promed. Revista Brasileira de Educação Médica, 32(3), 333-346.

Spaulding, W. B. (1969). The undergraduate medical curriculum (1969 model): McMaster university. Canadian Medical Association Journal, 100(14), 659.

de Camargo Ribeiro, L. R., \& Mizukami, M. D. G. N. (2004). Uma implementação da aprendizagem baseada em problemas (PBL) na pós-graduação em engenharia sob a ótica dos alunos. Semina: Ciências Sociais e Humanas, 25(1), 89-102.

Faculdade de Medicina de Marília. (2014). Projeto pedagógico do curso de medicina.

Komatsu, R. S., de Queiroz Padilha, R., \& Caleman, G. (2000). Interdisciplinaridade na educação médica: a experiência da Faculdade de Medicina de Marília (Famema). Revista de Administração Pública, 34(6), 81-93.

Depresbiteris, L. (2001). Certificação de competências: a necessidade de avançar numa perspectiva formativa. Formação, 2, 27-38. 
Perrenoud, P. (1999). Avaliação: da excelência à regulação das aprendizagens-entre duas lógicas. In Avaliação: da excelência à regulação das aprendizagens-entre duas lógicas (pp. 183-183).

Reid, J. A. (2011). Understanding Medical Education: Evidence, Theory and Practice, 1st edn.

Minayo, M. C. D. S., Deslandes, S. F., \& Gomes, R. (2016). Pesquisa social: teoria, método e criatividade.

Komatsu, R. S. (1999). Aprendizagem baseada em problemas: um caminho para a transformação curricular. Revista Brasileira de Educação Médica, 23(2-3), 32-37.

Zanolli, M. B., Boshuizen, H. P., \& De Grave, W. S. (2002). Students' and tutors' perceptions of problems in PBL tutorial groups at a Brazilian medical school. EDUCATION FOR HEALTHABINGDON-CARFAX PUBLISHING LIMITED-, 15(2), 189-201.

Faria, M. J. S. S. D., Nunes, E. D. F. P. D. A., Anastasiou, L., Sakai, M. H., \& Silva, V. L. M. D. (2008). Os desafios da educação permanente: a experiência do curso de Medicina da Universidade Estadual de Londrina. Revista Brasileira de Educação Médica, 32(2), 248-253.

Moia, L. D. J. M. P., de Sousa, R. P. M., Souza, R. M. V., \& da Fonseca, A. B. (2017). Metodologias ativas de ensino-aprendizagem: perfil e capacitação pedagógica do docente do curso de medicina. IJHE-Interdisciplinary Journal of Health Education, 2(1).

Barreto, N. A. P., Xavier, A. R. E. D. O., \& Sonzogno, M. C. (2017). Percepção de tutores quanto a sua avaliação pelos discentes de um curso médico. Revista Brasileira de Educação Médica, 41(2), 221-230.

Seren, S., \& Ustun, B. (2008). Conflict resolution skills of nursing students in problem-based compared to conventional curricula. Nurse Education Today, 28(4), 393-400. 